\title{
LE ROI EST MORT! VIVE LE ROI?
}

Unias ARANTES ${ }^{1}$

- RÉSUMÉ: Ce texte examine critiquement les points d'appui et propos philosophiques du projet culturel néomoderne (Habermas, Roùanet), interroge le lien avoué entre modernité et néomodernisme, le débat de celui-ci avec les irrationalismes post-modernes, explicite préssuppositions et inconsistances du projet néomoderne ainsi que ce que le néomodernisme laisse dans l'ombre dans son rachat de la modernité et ce que par conséquent il ne peut pas penser. Sous le fil conducteur de la critique du néomodernisme, on a souligné une sorte de "fuite en avant" qui serait le destin commun des intellectuels brésiliens, la possibilité et la limite de leur existence sociale.

- MOT-CLÉFS: Modernité; Illustration; néomodernisme; post-modernité; irrationalisme; raison systémique; rationalité communicative; emancipation.

\section{Néomodernisme}

Le diagnostic est celui d'une crise: crise de la modernité; ou, ce qui semble revenir au même, crise de la raison. L'irrationalisme n'est certes pas la cause de la crise, mais plutôt comme une mauvaise reaction à la crise. Une sorte de médicament qu'au lieu de guérir de la maladie, tue le malade. S'il est un mauvais traitment c'est parce qu'il est fondé également sur un mauvais diagnostic. En effet, l'irrationalisme mécomprend la crise, la renforce et finit presque par se confondre avec elle. Pour les combattre - pour combattre correctement les deux, la crise de la modernité ou de la raison et l'irrationalisme - la seule voie est le néomodernisme. Une thérapie analogue en quelque sorte à la homéopathie: similia similibus curantur. L'irrationalisme, au contraire, jette le bébé avec l'eau du bain.

Le néomodernisme que propose S. P. Rouanet (1987) est pour l'instant une programme à être accompli, mais dont on peut déjà connaïtre les trois étapes. Il faudra

1. Prof. Dr. Urias Arantes (Programa de Pós-Graduação em Filosofia da PUC/SP. Pesquisador ligado ao Colégio Internacional de Filosofia - Paris). 
d'abord relever les "élements structurels" de l'illuminisme, de la tendance intellectuelle transhistorique à combattre le mythe et le pouvoir avec les armes de la raison. Il faudra ensuite donner à l'illuminisme une base normative, un ensemble de valeurs au nom desquels combattre les distortions du présent. Il faudra enfin que le projet soit soumis à l'épreuve de la réalité pour montrer qu'il trouve un repondant matériel dans les tendances présentes de la société.

La nouvelle figure de l'illuminisme reprend à la plus importante de ses manifestations antérieures, l'illustration, son noyau dur, à savoir, le projet d'émancipation tel qu'il s'exprime dans le mot d'ordre de l'Aufklarung, selon Kant: Sapere aude! Cet élément historique comporte deux vecteurs, la critique et la raison, c'est à dire, la critique de toutes les tutelles à partir de la raison. Le néomodernisme cependant n'a affaire ni à la même raison ni à la même critique. Au XVIII siècle la raison ignorait les limites intérieures et extérieures de la rationalité. Elle ne distinguait pas entre raison et idéologie. La nouvelle raison se doit d'être capable de critique, mais aussi d'auto-critique, et d'être armée pour démasquer les discours où l'irrationnel opère sous l'apparence de la rationalité. La critique est par ailleurs toujours critique de l'actualité, ce qui voulait dire, au XVIII siècle, combattre les conditions structurelles qui interdisaient l'autonomie. Or, aujourd'hui, l'autonomie est largement acquise, grâce justement à l'illustration. Seulement ces progrès ont engendré des nouvelles formes d'hétéronomie qu'il faut maintenant combattre.

Soulignons seulement, avant de passer avant, qu'il y a là un point crucial: un projet d'autonomie engendre de l'hétéronomie. Le néomodernisme se donnera-t-il les moyens critiques de rendre compréhensible cette inversion?

Mais la critique et l'auto-critique n'est rationnelle que si leur travail s'appuie sur un fondement "normatif", sous peine de s'avérer "aveugle, anarchique et nihiliste". Ce fondement, le néomodẹrnisme le trouve déjà présent dans l'illustration, sous la forme des idéaux du XVIII siècle éclairé. Il suffit de les adapter, voire d'élargir selon les exigences d'aujourd'hui. Ainsi, par exemple, la nouvelle raison se laisse orienter par le pluralisme et la tolérance, sans toutefois ignorer le combat d'aujourd'hui: il serait moins contre l'église et les tyrans que contre les mécanismes sociaux et psychologiques. Ainsi le progrès reste encore une valeur fondatrice, mais elle ne s'appuie plus sur une philosophie de l'histoire: la progrès est conçue maintenant comme contingent et dépendant de l'action consciente des hommes.

Si nous nous souvenons ici du point crucial souligné avant, nous trouverons un premier élément de réponse. Les fondements de l'illustration et du néomodernisme sont essentiellement les mêmes. Il s'ensuit que l'hétéronomie engendrée par la première ne s'explique pas par les fondements du projet d'autonomie de l'illustration, et par conséquent elle serait en quelque sorte sécondaire. Une simple déviation? Voyons si la suite confirme ou non ce début de réponse.

Ne peut-on douter par ailleurs que le beau projet du néomodernisme ne soit plus qu'une Schwärmerei? Ca serait ignorer, selon lui, ce que les analyses de Habermas ont 
mis en lumière: l'existence d'un illuminisme spontané, pré-réflexif, agissant dans les structures de la communication quotidiènne. Tout y est déjà en herbe: la raison qui propose et conteste des arguments, la critique qui dénonce la volonté de pouvoir, le patrimoine des valeurs illuministes actualisées lors du processus communicatif: l'homme est capable de savoir et de véracité, de justice et d'autonomie, de dialogue et de compréhension réciproque. Le néomodernisme est ainsi une construction théorique qui dispose d'un "sol social", car il est rélié à l'illuminisme spontané.

Le projet néomoderne n'est pas achevé, et d'ailleurs la tâche est collective. Étant donné l'ébauche de ce qu'il y a à faire, il est facile d'imaginer que le maître de chantier s'appelle Habermas. Ce qui nest pas encore une critique, mais seulement un constat.

\section{Les nouveaux barbares}

La nouvelle forteresse théorique bien plantée sur le social et sur la tradition se dresse d'abord contre les attaques (irrationnels?) de l'irrationalisme en trois fronts: la raison, la modernité, l'illustration. À cela s'opposent trois opérations défensives: le rachat critique du concept de raison, le projet de la modernite et l'héritage de l'illustration. L'on voit bien qu'il ne s'agit pas de sauvegarder à n'importe quel prix la raison, la modernité et l'illustration. Car la crise culturelle contemporaine n'est pas une invention de l'irrationalisme. Seulement celui-ci dans sa démesure la prend comme preuve d'un échec et donne manifestation d'un besoin de passer à quelque chose d'autre, à un au-delà ou en-deça de la raison. Or, ce désir d'un nouveau absolu est en réalité réactionnaire, mais non pas de la même façon des ennemis autrefois de l'illustration. Contre des nouveaux ennemis, des nouveaux remèdes. Et les ennemis ne manquet pas: la vieille droite pour qui le néomodernisme dissout les valeurs traditionelles; les libéraux et conservateurs modernes qui ne conçoivent plus de critique globale; la gauche classique que n'y verra qu'un avatar de l'idéologie bourgeoise; et la nouvelle gauche irrationaliste, car pour elle toute raison est dictatoriale et répressive. Les nouveaux barbares sont en réalité ces derniers, les plus dangereux.

Nouveaux d'abord parce qu'ils sont critiques et non pas conservateurs (comme s'il ne pouvait pas avoir une critique conservatrice!). Essentiellement ils dénoncent le compromis de la raison avec le pouvoir pour opprimer la vie. Ils s'appelent Foucault, Deleuze, Lyotard. La critique de la nouvelle gauche ne s'est pas complètement trompée: il faut une revision du concept classique de raison. Revision, et non pas démolition de son noyau dur: la raison seule est critique, car elle seule peut dépasser la facilité par et dans le travail du concept. C'est par là que l'irrationalisme est conformiste: il resterait au ras des pâquerettes de par son refus du concept.

La nouvelle raison, critique et auto-critique à la fois, reconnaît et critique l'irrationalité présente dans le sujet cognitif lui-même et dans les institutions extérieures. Le paradigme critique a été explicité par Habermas: au lieu de la relation 
classique Sujet-Objet, la relation communicative en vue d'un consensus impliquant trois contextes différents: le monde objectif des choses, le monde social des normes et le monde subjectif du vécu. La rationalité s'y manifeste: "seront rationelles non pas les propositions correspondantes à une vérité objective, mais celles validés dans un processus argumentatif où le consensus a été obtenu sans déformations extérieures résultant de la violence, sans déformations intérieures résultant de la fausse conscience, à travers preuves et contre-preuves, arguments et contre-arguments'" (p. 14).

Ce nouveau paradigme s'impose à partir de la modernité elle-même dans la mesure où elle posait l'émancipation en termes d'autonomie à l'égard de la tradition et de l'autorité. Il est vrai que la modernité a engendré en même temps d'autres formes d'hétéronomie, particulièrement la "raison instrumentale" qui coordonne automatiquement les individus dans la sphère systémique de l'état et de l'économie. La modernité a en fait créé deux rationalités: la systémique et la communicative. Il est juste de combattre la raison instrumentale, mais cette critique ne peut se faire qu'au nom de la raison critique, sans quoi elle tombe dans l'irrationalisme. Celui-ci se trompe parce qu'il confond raison systémique et raison-en-soi, une distinction qui est d'ailleurs à la base départ de la théorie habermasienne.

Le soupçon de réponse que nous avions trouvé avant, à savoir que le projet d'autonomie de la modernité engendrait de l'hétéronomie sans pour autant en être affecté essentiellement, semble être quelque peu confirmé ici, dans cette idée de la double rationalité. Les nouveaux barbares se trompent parce qu'il s'attaquent à l'inessentiel (qu'ils confondent avec l'essentiel). Même si l'on ne s'étonne pas de l'expression "deux raisons", il est difficile de ne pas revenir à las questions des eventuels rapports et implications entre les deux. La question demeure entière: comment le projet d'autonomie engendre de l'hétéronomie? Il n'y a donc pas une intime complicité entre "raison systémique" et "raison-en-soi"?

Ceux qui dénient la raison ou plutôt qui confondent les deux, ceux-là se prétendent des "post-modernes". Le réfus de la modernité ou la reconnaissance de son échec se fait dans chacune de ses articulations essentielles: l'économie, la politique, la science, la philosophie, l'esthétique. L'économie moderne est morte avec l'industrialisation qui cède la place aux sociétés de service; la politique moderne se fondant sur la représentation et sur le jeu des partis, elle n'a plus de sens dans un espace public fragmenté par un reseau de micro-mouvements s'opposant à des micro-pouvoirs; la science moderne fondé sur le "grand récit" disparait devant des savoirs paralogiques et le pragmatisme inhérent à l'activité scientifique elle-même; la post-modernité philosophique abandonne elle aussi les "grands récits" pour s' occuper de défaire toutes les illusions de la modernité; l'art post-moderne enfin se nourrit de l'épuisement des paradigmes et fait recours à la citation et au pastiche.

Or, argumente le projet néomoderne, cette condition post-moderne n'est pas une rupture d'avec la modernité, mais plutôt des manifestations de ce qui s'y trouvait déjà. (Lyotard, d'ailleurs, ne dit pas autre chose lorsqu'il explique le post-moderne aux 
enfants.) D'où l'inutilité d'un concept nouveau pour en parler. Le dépassement de l'industrialisation était inscrit dans la logique du capitalisme. L'apparition des nouveaux droits était rendu possible par la doctrine moderne des droits de l'homme. La validation des énoncés scientifiques n'est pas une nouveauté, car elle tient toujours à l'acceptation par la communauté scientifique. En philosophie, la critique de la modernité et de ses illusions est contemporaine à la naissance de la modernité. L'art enfin n'a rien perdu de son autonomie. Ceci étant, parler de post-modernisme c'est confondre le désir de rupture avec l'événement de la rupture. La critique post-moderne de la modernité est justifiée, mais elle ignore que la modernité est ambivalente. Il suffit de s'appuyer sur son "vecteur d'émancipation" pour faire ressortir une modernité plus libre, la néomodernité qui n'est rien d'autre que la présence active de la raison "sage" au coeur même de ce mélange de sagesse et folie que nous appelons "modernité". La modernité ainsi comporte ses propres instruments d'émancipation: une raison autonome (capable de dénoncer la raison systémique), une action morale autodéterminée (independante des autorités extérieures) et une politique consciente (fondée sur des structures démocratiques). Les post-modernes prennent la modernité comme un projet qui a échoué; les néomodernes, comme un projet inachevé.

Il reste à s'interroger sur l'héritage de l'illustration. Il y a certes des promesses qu'elle n'a pas honorées, ou dont les réalisations ont été desastreuses: la croyance dans le progrès a ouvert la voie à toutes les répressions; l'individualisme a engendré le sujet égoïste; l'utopie de la révolution sociale a produit l'utopie concentrationnaire; le rêve de l'autonomie a laissé l'homme solitaire sous un ciel vide. Cependant l'illustration n'était que la reprise d'une impulsion critique et renovatrice beaucoup plus grande et qu'elle n'a pas épuisé. L'illustration ne se confond pas avec l'illuminisme: le combat contre le mythe et le pouvoir à partir de la raison. En ce sens, la critique de l'illustration est en même temps une auto-critique d'où il ressortira l'héritage positif de celle-là pour constituer le fond de l'illuminisme moderne.

Ce bilan des promesses non honorées a quelque chose de troublant car il semble mettre à côté de chaque trait distinctif de la "raison-en-soi" la contrepartie de la "raison systémique". Il est alors impossible de penser à une simple déviation. L'ambivalence s'avère être une profonde ambiguité par-dessus laquelle saute allègrement le projet néomoderne. La cause peut-être en est qu'a prendre cette question par les cornes le risque est grand de ne pouvoir parler ni de projet ni de néomodernité.

\section{L'enthousiasme du pédagogue ou le complèxe du castor}

J. A. Giannotti (1987) a tout à fait raison de demander à S. P. Rouanet un "filet catégoriel plus fin", malgré la concession à une certaine facilité que commande sa critique. Examiné avec un peu plus d'exigence au niveau de ses articulations 
principales, le projet néomoderne - ou plutôt le projet du projet - s'avère d'une charpente peu solide que le registre d'exposition ne suffit pas à justifier. D'autant plus que la faiblesse tient à ce que l'exposé laisse dans l'ombre. C'est cette exclusion, cet oubli qui rendent possible le projet tel qu'il s'énonce; ils témoignent d'un point aveugle constitutif. Il convient de se demander si la critique n'est pas elle-aussi atteinte.

Le fondement du projet néomoderne est en dernière analyse la transhistoricité de l'illuminisme, l'illustration ayant été sa manifestation historique la plus importante, mais non pas la seule. Pensons par exemple à Erasme. L'exemple d'ailleurs est donné par Rouanet lui-même. Il y voit, entre autres choses, dans les rapports entre Erasme et Luther, un nouvel épisode de la guerre entre l'ombre et la lumière. Malheureusement pour lui, dans les affaires humaines il faut plutôt faire dans la dentelle, comme Kant (et pour cette affaire en particulier, Weber) aurait pu lui apprendre.

La transhistoricité de l'illuminisme (et la raison communicative habermasienne qui en semble être la confirmation) a une sorte d'anthropologie implicite, tout au moins l'affirmation d'une distinction prope à l'humanité qui est beaucoup plus optimiste que celle de Kant. La comparaison ici n'est pas du tout aléatoire, les héritiers néomodernes voyant chez Kant le plus grand de ses testateurs. L'anthropologie kantienne, par ailleurs, est conçue comme une connaissance théorique: conaissance de la nature humaine. Ce qui soulevait la question complexe - et pour Kant sans réponse définitive - du passage (Übergang) de la nature à la morale, du théorique au pratique, dont un dédoublement important, sinon essential, comme nous l'avons vu, est certainement la réflexion sur l'histoire de l'humanité. Au contraire, l'anthropologie implicite dans le néomodernisme est une Idée, au sens kantien, qui se serait manifesté plus clairment à certains moments de l'histoire et qu'il s'agit de réactualiser maintenant sous la forme d'un nouveau programme culturel. La tâche principale étant de reconstruire le concept de raison et de lui donner valeur normative. Mais alors toute l'interrogation sur le "passage" de la nature à la morale, de l'humanité civilisée à l'humanité morale est laissée de côté. Même si l'on admet que Marx et Freud auraient développé une anthropologie théorique, ce qui ne semble pas être le cas. À la place de l'interrogation sur les passages, le project néomoderne semble énoncer un pari, au risque de ne faire qu'un voeu pieux.

Une anthropologie néomoderne se doit de rester implicite, car le "paradigme" de la rationalité n'est plus la relation Sujet-Objet de la modernité, mais la relation communicative. La rationalité se laisse désormais voir comme ensemble de règles du processus communicatif, tandis qu'avant elle était une faculté du Sujet (un don et une destination, selon Kant) dont le fondement exigeait une certaine conception de l'homme comme être capable de raison. Cependant si le nouveau paradigme ne semble plus avoir besoin du "grand récit" fondateur, il se doit de considérer l'action communicative exerçant les régles de la rationalité en vue d'un consensus comme un idéal, c'est à dire comme purifié de la violence et du jeu d'intérêts qui troublent la communication empirique. C'est par là que le project néomoderne devient un pari sur la rationalité communicative: "si nous gagnon, nous emporterons tout: si nous perdons, 
nous ne perdons rien, car nous ne pouvons pas devenir plus pauvres que nous le sommes déjà"' (p. 347). Je ne suis pas sûr qu'il y ait au point de départ ou au point d'arrivée de la pensée de Habermas un tournant pascalien, mais peu importe ici.

Je ne suis pas sûr non plus qu'il suffise d'énoncer les termes d'un pari pour rompre le raisonnement circulaire qui est à la base du projet néomoderne et que l'on pourrait formuler comme suit: la "nouvelle raison" est ce qui a toujours guidé sous d'autres formes l'humanité, il s'agit d'en faire l'horizon indépassable. Cette circularité est déjà en quelque sorte le mouvement dessiné par le geste critique kantien: remonter de ce on qui est à la condition de sa possibilité c'est reconnaître que le fondement dépasse le fondé pour lui désigner également son devoir-être, qui devient par lá destination originaire. On pourrait penser que le projet néomoderne repète à sa façon le geste critique de l'Aufklarung, au moins dans sa version kantienne.

Cependant il est clair que le geste kantien implique la reconnaisance d'une fracture, d'un manque constitutif d'unité au coeur même des actions et des institutions humaines; une fracture irréductible qui constitue la "nature" humaine et les rapports que les hommes nouent entre eux. C'est cette reconnaissance qui fait que, pour Kant, l'essentiel se joue dans la dimension pratique et que l'essentiel de l'essentiel se joue dans l'historico-politique. C'est là en fait l'espace d'une fracture en même temps intérieure et extérieure aux hommes. Si il y a un héritage des Lumières - et je suis convaincu qu'il en ait - à être recueilli et sauvegardé c'est peut-être alors celui de ce lieu fondamental d'interrogation, cet espace entre. Ce qui ne semble pas, par ailleurs, être dénié par ceux qui parlent d'inachèvement, d'échec ou de liquidation du projet moderne. J'aurai envie de dire que la "fin de la philosophie" est contemporaine de l'exigence "nouvelle" de la réflexion politique.

Or, c'est justement ce que le projet néomoderme laisse dans l'ombre dans son rachat de la modernité et ce que, par conséquent, il ne peut pas penser, si ce n'est que sous la forme d'un ensemble de valeur normatives à être promues. Par-là également le projet néomoderne s'interdit de formuler des questions centrales pour la compréhension de l'actualité: d'où vient-il qu'il y ait crise? En quel sens parle-t-on de crise de la modernité? Quelle raison s'identifie à quelle modernité? Les événements majeurs de la modernité (les totalitarismes, la possibilité d'une destruction totale, la fin des totalitarismes, l'identification de la démocratie au capitalisme etc.), quelle lumière lancent-ils sur le "projet" moderne? Le projet néomoderne parle de "l'ambivalence" de la raison moderne, comme si celle-ci avait dans sa réalisation engendré d'un côté du bon, de l'autre, du mauvais. Mais "bon" et "mauvais", " 'raison sage" et "raison folle" sont-elles seulement séparables?

Une dernière question: à quelle question le project néomoderne se veut comme réponse? La cecité au projet politique de la modernité, l'insensibilité à ses avatars, semble faire du projet néomoderne quelque chose comme le discours d'un pedagogue qui aurait scamoté l'histoire de ses auditeurs pour leur faire croire que le manque d'horizon historique est la faute de ceux qui n'en voient pas. Ou comme sait tout un chacun: les enfers sont pleins de bonnes intentions! 


\section{Ultra aequinoxialem non peccari}

Les combats rudes entre la raison et l'irrationalisme n'ont pas épargné les douces terres tropicales, preuve peut-être supplémentaire que l'humanité est la même à Prague, à Frankfurt ou à Rio. Cependant sous les tropiques tout est un peu plus contrasté (serait-ce un effet du trop de lumière?). C'est ainsi que la raison s'est vue ici soumise à deux logiques de la sphère systémique sous la forme de raison d'état et raison économique. Et soumise non pas comme résultat des ambivalences de la raison illustrée, mais plus grossièrement comme résultat de 20 ans de régime militaire. Les conséquences de cette entreprise autoritaire ont été si importantes qu'il n'y avait plus de place pour une raison alternative; et la critique de la dictadure n'a pas été possible que sous la forme d'une contestation de la raison elle-même. Tiens, voilà que le Cebrap, certains secteurs de l'église catholique, les différents mouvements ouvriers, ou alors les mouvements d'opinion pour les élections directes - eh oui, de l'irrationalisme! Gauchisme conservateur et irrationel! Justifiés, certes, par l'identification autoritaire opérée par la dictadure entre raison et sphère systémique. La dictadure et ses opposants deviènnent ainsi curieusement complices, car lorsque le processus de démocratisation a commencé - c'est à dire, lorsque le "processus communicatif libre" pouvait être "repris" - la raison était déjà identifiée à l'économie. L'analysis objective devint impossible, les reactions émotionnelles predominèrent conduisant au retour de prototypes anciens. La raison était devenu l'ennemie à abâttre: ennemie de la vie, de la spontanéité populaire, des les revendications ouvrières, de l'art, de l'autenticité nationale. La pratique seule comptait, car en elle vit la vérité. Il y a ainsi, au Brésil, un arrière, la remise en circulation d'un débat des années 20 et 30 en Allemagne, le romantisme allemand ou française ou un ISEB de caricature.

Une métaphore psychanalytique, nous suggère-t-on, aide à mieux comprendre ce qui s'est passé au Brésil ces dernières decennies: la notion freudienne de "période de latence" (Latenzperiode): une période d'oubli (située entre le déclin de l'Oedipe et la puberté) partiel et d'impossibilité d'accepter la nouveauté. Voilà une métaphore du Brésil sous le gouvernement autoritaire. Oubli ou refoulement? C'est peut-être sécondaire pour le sens de la métaphore. L'important c'est que pendant cette période nous n'avons rien appris, nous sommes restés virtuellement hors de l'histoire, et que nous avons conservé l'obscur souvenir des thèmes d'avant 1964. La période de latence terminée, ces thèmes remontent et sont traités comme des découvertes inédites, en géneral sans le raffinement théorique original.

Il serait amusant de poursuivre la métaphore. Par exemple, en amont on pourrait bien se demander à quoi corresponderait l'impossibilité inteme de satisfaire l'Oedipe qui accompagne l'entrée dans la période de latence. Étions nous avant 1964 épris d'un amour sans espoir? Le coup d'état aurait-il fonctionné comme un rappel au principe de réalité? Je laisse au lecteur le plaisir d'imaginer les question en aval, du coté de "l'ouverture politique" comprise comme résultant de la pression pubertaire! 
Ce qui frappe même le lecteur le mieux disposé, c'est la simplicité excessive avec laquelle Rouanet donne un sens aux dernières années de l'histoire brésilienne, pour l'inscrire dans le grand combat de l'humanité. C'est comme si nous avions parcouru en 20 ans ce que l'Europe aura mis deux siécles à accomplir. J'exagère sans doute, mais comment ne pas le faire quand on lit que notre passé récent (si l'on peut appeler passé) tombe sous la même grille analytique européene depuis le XVIII siècle, et même avant, car il ne faut pas oublier Thomas Morus, cet illuministe avant la lèttre.

Tout se passe comme si les questions qui cherchent à s'élaborer venaient toujours déjà de ce qui est pensé et en quelque sorte maîtrisé. Les questions sont alors énoncées en termes de manque ou défaut de théorie, manque ou défaut de concept. Par conséquent la réponse se laissera lire dans la suite du jeu conceptuel. La pensé se donne par là l'assurance douteuse de se mouvoir toujours en terrain ferme, où la confrontation avec son autre est d'emblée exclue. Dans l'espace propre du jeu de concepts, le "réel" ne constitue pas un danger, car il est toujours déjà pris dans du pensé ou dans du pensable. Il n'y a pas de vide, Il n'y a pas de manque. La pensé est alors de l'ordre de l'omnipuissance. En demandant a Rouanet un "filet catégoriel plus fin", j'ai le sentiment que Giannotti partage cette illusion originaire, et qu'il la reconforte. Ne serait-elle pas plus partagé que l'on ne croit?

\section{Fuite en avant}

Et pourtant, pour Giannotti, le roi semble bel et bien mort, un simple lifting ne suffisant pas à restaurer l'illusion de son éternelle jeunesse. Car sa mort est apparemment moins l'effet d'une maladie que le résultat d'une sorte d'éclatement de son pouvoir en autant d'espaces qu'il y a des "systèmes" de langage. Lyotard se sert de la métaphore de l'archipel, mais c'est une autre histoire qu'il ne convient pas d'aborder ici.

Ce que je voulais souligner c'est plutôt une sorte de fuite en avant qui est le destin commun des intellectuels brésiliens: la possibilité et la limite de notre existence sociale. Il faut toujours trouver le truc qui marche, l'articulation conceptuelle qui permette d'expliquer et de maîtriser le monde; il nous faut surtout que cela nous renforce dans la croyance en notre pouvoir de représenter la maison que l'architecte a en tête avant que les ouvriers se mettent à la construire. Nous avons des réponses trop des réponses. Mais ce n'est pas parce que nous avons oublié les questions?

ARANTES, U. O rei está morto! Viva o rei? Trans/Form/Ação, São Paulo, v. 15, p. 113 - 122, 1992.

- RESUMO: Este texto examina criticamente pontos de apoio e propósitos filosóficos do projeto cultural neomoderno (Habermas, Rouanet), interroga o vínculo confesso entre neomodernismo e modemidade, 
o debate deste com os irracionalismos pós-modernos, explicita pressupostos e inconsistências do projeto neomoderno, assim como aquilo que o neomodernismo deixa na sombra em seu resgate da modemidade e que, por conseqüência, não pode pensar. Sob o fio condutor da crítica ao neomodemismo de Rouanet, sublinha-se uma espécie de fuite en avant que seria o destino comum dos intelectuais brasileiros, a possibilidade e o limite de sua existência social.

- UNITERMOS: Modernidade; ilustração; neomodemismo; pós-modemidade; irracionalismo; razão sistêmica; racionalidade comunicativa; emancipação.

\section{Référence bibliografique}

GIANNOTTI, J. A. O tema da ilustração em três registros. Novos Estudos Cebrap, São Paulo, n. 18 , set. 1987.

ROUANET, S. P. As razões do Iluminismo. São Paulo: Companhia das Letras, 1987. 